\title{
Microprocessor software applications in color graphics
}

\author{
FRED RODGERS \\ Trinity University, San Antonio, Texas 78284
}

\begin{abstract}
This paper considers software capabilities and limitations relative to the development of a microprocessor-based operating system for the Intecolor $8001 \mathrm{G}$ graphic terminal. Complete discussions of the implementation of a microoperating system are included, from initial design considerations to descriptions of techniques used in the final system.
\end{abstract}

Traditionally, color graphic display systems have been prohibitively expensive for all but the most sophisticated users. Two major factors contributing to the high cost of these systems are (1) memory required for the display (about $128 \mathrm{~K}$ bytes for a 512 by 512 element, eight-color display), and (2) specialized processing hardware necessary for display generation. Technological advances made in the electronics industry are having a dramatic effect on the cost and performance of components used in such systems. Memory with a cost of .5 cents/bit in 1974 currently costs .05 cents/bit, and this is estimated to be .01 cents/bit by 1981 . The result of this decline in cost is that $128 \mathrm{~K}$ bytes of memory, valued at more than $\$ 5,000$ in 1974 , now cost little more than $\$ 500$ and are projected to be available for $\$ 100$ in 1981. Even as memory prices have gone down, performance has continued to increase. Typical access times are projected to drop from $400 \mathrm{nsec}$ in 1974 to less than $200 \mathrm{nsec}$ by the 1980s (Rao \& Hewkin, 1978).

The many digital logic circuits once necessary in any sophisticated color graphic display system have been replaced by a single integrated circuit, a microprocessor. The development of microprocessor chips, which has occurred entirely within the last 10 years, is having a profound effect on almost all aspects of computing, especially color graphic display systems. Performance increases and cost decreases of microprocessors are comparable to those of memory chips, but it is difficult to make any quantitative comparisons because the capabilities of the microprocessors have changed so significantly over the years. Early microprocessors had only a few 8-bit registers, could address only 16,000 bytes of memory, had very limited instruction sets, and had cycle times of only $1 \mathrm{MHz}$. Today's microprocessors have 16 general-purpose 16-bit registers, can address 16 million bytes of memory, have very extensive and powerful instruction sets, and have cycle times of 4 or $5 \mathrm{MHz}$ (Intel Corporation, 1978).

Partial support for this project was made available through cost-sharing RULE and ISEP grants from the National Science Foundation, SER 76-14507 and SER7 7-10916. Requests for reprints, information, and source program listings should be addressed to Fred Rodgers, Computer Center, Trinity University, 715 Stadium Drive, San Antonio, Texas 78284 .
Since hardware costs are becoming a minor limiting factor in color graphic systems, a much more significant problem is software. Full utilization of microprocessor capabilities to provide easier and more effective use of computing devices is a challenge that we have just begun to meet. This paper describes the development of a user-oriented microprocessor-based operating system for the Intecolor $8001 \mathrm{G}$ graphic display terminal. This system has attempted to take advantage of the capabilities of the microprocessor in order to provide host compatibility, distribution of processing and simplification of command structures.

\section{HARDWARE OVERVIEW}

Electronic hardware found in the Intecolor $8001 \mathrm{G}$ is of two types, digital and video (see Figure 1). The video circuitry is similar to that found in standard raster-scan color televisions, the difference being that the scan rate has been significantly increased to provide higher resolution. The video signal is therefore incompatible with standard video signals and cannot be used with conventional color video monitors and recorders.

Intecolor digital hardware consists of the central processing unit (CPU), memory, and screen display generator. The $\mathrm{CPU}$ assembly contains a $2-\mathrm{MHz}$ 8080A microprocessor, a TMS5501 multifunction $\mathrm{I} / \mathrm{O}$ controller (Osborne, 1976), and eraseable programmable read-only memory (EPROM). System software, stored

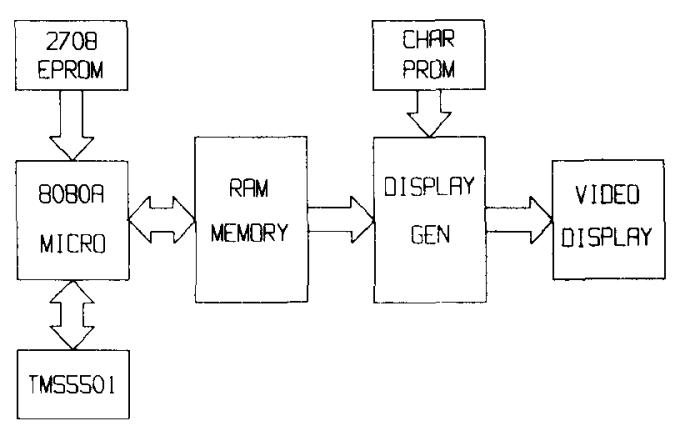

Figure 1. In tecolor hardware overview. 
in EPROM and executed by the microprocessor, gives the terminal its "intelligence." The TMS5501 provides the microprocessor with such features as serial and parallel communications interfaces, a priority interrupt structure, and interval timers. The system memory is provided by an Intel 2107B-4 equivalent (Intel Corporation, 1977). These 4K-bit chips are organized into 8K-byte boards, with a maximum of four boards (32K bytes) per system. A dedicated area of randomaccess memory (RAM) is continually scanned by the screen display generator, using a direct memory access (DMA) technique. This information is converted in to display dot patterns using character generator PROMs (see Figure 2). The dot patterns are passed to the video hardware, where they are finally displayed on the screen (Intelligent Systems Corporation, 1976).

The screen is organized into 48 lines of 80 characters; 2 bytes in RAM correspond to each of these 3,840 positions. The first byte contains the actual character to be displayed; the second contains color, blink, and plot information. In character mode, the 64 standard ASCII characters occupy Positions 20 to 5F hex; 00 to $1 \mathrm{~F}$ hex and 60 to $7 \mathrm{~F}$ hex are reserved for 64 special characters. Values from 80 to FF hex indicate double character height, where the top or bottom half of the character is displayed as a full character, depending on whether or not it is on an odd or even line (see Table 1). Graphic displays are created by turning on the plot bit that indicates that special plot generator PROMs are to be used instead of standard ASCII character PROMs (see Figure 3). In graphics mode, each character is divided into eight elements, four high and two wide, giving an overall screen resolution of 192 by 160 elements (Dwyer \& Critchfield, 1978). While the graphic resolution of the display is 192 by 160 points, the color resolution is only 48 by 80 . This can create significant problems when doing high-resolution multicolor displays.

\section{THE INTECOLOR 8001G IN AN IBM 370/APL ENVIRONMENT}

APL, a programming language (Gilman \& Rose, 1974), is an interactive timesharing terminal system highly oriented to mathematical matrix manipulations and therefore well suited to color graphics applications. Since considerable graphic software had already been developed for such devices as the Tektronix 4013 (Tektronix, Inc., 1973), it was desirable to connect

Table 1

Character Code Ranges

\begin{tabular}{lcc}
\hline Plot Bit On & 256 Plot Characters & $00-\mathrm{FF}$ \\
Plot Bit Off & 32 Special Characters & $00-1 \mathrm{~F}$ \\
& 64 ASCII Characters & $20-5 \mathrm{~F}$ \\
& 32 Special Characters & $60-7 \mathrm{~F}$ \\
& 128 Characters* & $80-\mathrm{FF}$ \\
\hline
\end{tabular}

* Same as above but double height.

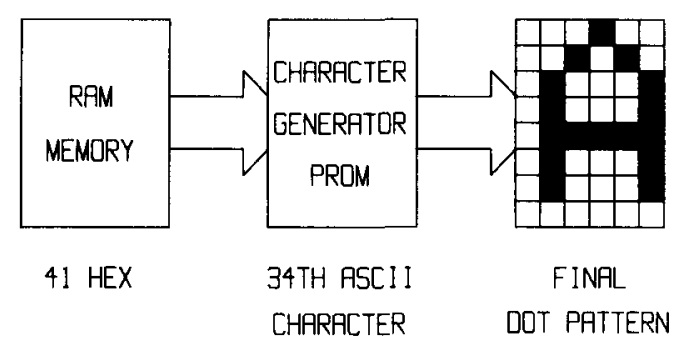

Figure 2. Dot pattern generation.

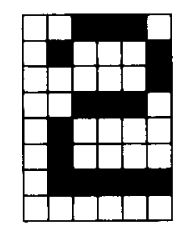

32 HEX

ASCII CHARACTER

PROM

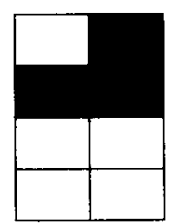

32 HEX

PLOT CHARACTER

PROM
Figure 3. Character and plot mode resolution.

the Intecolor terminals to the APL system. However, the following problems required solution before such an interface could be considered successful.

\section{Basic Communications Discipline}

APL terminals use standard ASCII codes (128 values, 00 to $7 \mathrm{~F}$ hex). These are transmitted as 10-bit characters: start bit, 7 data bits, parity bit, and stop bit. By sending an 8th data bit instead of parity, the Intecolor uses 256 values ( 00 to $\mathrm{FF}$ hex). Intecolor graphic coordinate data values are expected in 1 byte with values ranging from 0 to 191. Data in this form are impossible to send with a 7-bit code and, even though the Intecolor had provisions for sending coordinate data using a 7-bit code, this was never successfully accomplished using the APL system. Nonstandard uses of various control codes caused considerable difficulty in working with the APL system. In any communications discipline, standardization of control codes between terminal and host computer is an absolute necessity.

\section{Intecolor 8001G System Timing Considerations}

In a timesharing environment such as APL, exact timing is impossible. Several Intecolor graphic functions require a minimum time delay for processing. Drawing a 100-element vector (about halfway across the screen) requires $34 \mathrm{msec}$ before the processor can accept more data. At 2,400 baud, eight characters can be lost while the processor draws a 100-element vector. Unless the host computer program calculates appropriate timing delays, data are lost and results are unpredictable. It was hoped that these problems could be solved by substantially lowering the baud rate or inserting idle characters, or both. Neither solution alleviated the 
timing problems, and both usually significantly degraded the performance of the graphics.

\section{APL Character Set}

The APL language makes extensive use of various Greek symbols to represent mathematical operators. Some operators are constructed by overstriking several of these symbols. The Intecolor $8001 \mathrm{G}$ character sets did not include any of the special APL symbols and had no facility for handling the overstriking of multiple characters.

\section{General Graphics Capabilities}

The Intecolor $8001 \mathrm{G}$ has a complex graphic plot mode structure (see Table 2). Use of these graphics functions from APL was difficult, and often, it was impossible to determine whether a particular program was not working because of timing problems, problems with coordinate data, or misunderstanding of the documentation manual. Because of existing software and development overhead, it became desirable to have a more standard graphics command format, such as that used on the Tektronix 4013 (Tektronix, Inc., 1973).

\section{A NEW OPERATING SYSTEM?}

Initially, only modifications to the original system were considered. Object code was read from ROM and disassembled. Source listings of the operating system were obtained, but they had incomplete documentation and did not exactly correspond to the purchased version of the system. It soon became clear that major modifications to the original system were necessary. $1 / O$ routines needed to be modified to conform to a new communications discipline; all of the graphic routines needed to be modified to accept a new format of coordinate data. Timing problems could be

Table 2

Intecolor Plot Command Summary

General plot mode

Character plot mode

Point plot modes

$\mathrm{X}$ point plot mode

$Y$ point plot mode

incremental point plot mode

Bar graph modes

$\mathrm{X}$ bar graphs

$\mathrm{X}$ value mode

$Y$ value mode

$\mathrm{X}$ max value mode

incremental mode

$\mathrm{Y}$ bar graphs

$Y$ value mode

$\mathrm{X}$ value mode

$Y$ max value mode

incremental mode

Vector plot modes

$X$ value mode

$Y$ value mode

incremental vector mode solved by buffering all input, and this would require additional memory, as well as major modifications to operating system $\mathrm{I} / \mathrm{O}$ routines. APL characters could be designed and burned into PROM, but overstrike recognition would require extensive programming changes. Simplification of the many plot modes was desirable, and it was hoped that by rewriting graphic subroutines, substantial performance improvements would be possible.

Since almost every aspect of the old operating system seemed to need major revisions, it was decided to completely rewrite the entire system. A more powerful and flexible operating system could be created by incorporating certain concepts throughout the basic design. These concepts are as follows: (1) Standard ASCII communications discipline-This allows communication with the APL system as well as most other systems. (2) Fully buffered 1/O-Commands from the host computer are stored in RAM memory until the processor has time to handle them. (3) Multiple character set support, including overstrikes-This allows terminals to be used as full APL terminals or as standard ASCII terminals; character sets are changeable under program control. (4) Simplification of graphics commands-All plot modes are combined in to basically two modes, point plot mode and vector plot mode. (5) Extension of graphic capabilities-By providing the user with a more powerful set of graphic commands, such as object definition, real-time object movement, and area shading, the power and usefulness of these terminals can be greatly increased.

\section{THE NEW SYSTEM}

Sof tware for the system consists of four main modules: (1) SYSIO-system I/O support routines including interrupt-driven buffering routines; (2) SYSSD-system screen display routines responsible for controling alphanumeric display of data; (3) SYSPL-system plot routines providing all gra phic support;(4) SYSTC-system telecommunications drivers providing basic communications support as well as recognition and execution of system command sequences.

System I/O routines (SYSIO) handle parallel data from the terminal keyboard and serial data coming from the host over a RS232 line. Incoming data, captured by the TMS5501, create a processor interrupt, and program control is passed to the appropriate device interrupt routine. If data are from the keyboard, the keyboard mode is checked for ASCII or APL key positions, and input is translated into the appropriate codes. Data are then placed in an $\mathrm{I} / \mathrm{O}$ buffer, pointers and counters are updated, and control is returned to the point before the interrupt occurs. The overhead involved in these interrupt routines is very small. By handling incoming data in this fashion, the processor is free to finish previous commands before processing new information, thus completely eliminating the need for any timing delay considerations. 
Screen display routines (SYSSD) are responsible for alphanumeric display formatting, including cursor positioning through such control codes as backspace, linefeed, carriage return, and horizontal and vertical tabulation. Display modes such as page mode (filling the screen from top to bottom) and scroll mode (single line at the bottom of the screen that rolls up as the display progresses), as well as character height and page erasure, are controlled by these routines. Either APL or standard ASCII characters may be used. In APL mode, character overstriking has been accomplished by defining all legal overstrike characters and burning them into PROM. Before a character is stored in the screen display, each position is checked to see if it is blank. If not, tables of valid overstrike characters are consulted and either the resulting overstrike or an error indicator is displayed.

System plot functions (SYSPL) have been simplified into two modes: point plot and vector plot. In point plot mode, a single element is displayed corresponding to a set of $X-Y$ coordinates. Elements approximating straight lines between pairs of $X-Y$ coordinates are used in vector plot mode. The algorithm used to generate these lines is highly table driven. Once the initial position and line direction are determined, a set of tables provides the necessary information to create the line. It was hoped that this table-driven method would be much faster, but while it was somewhat faster, no significant improvements were realized. This is probably attributable to the limited number of registers available on the $8080 \mathrm{~A}$ microprocessor. Because of the register limitations, table look-up overhead was greater than anticipated.

Since color resolution is not as great as the graphic element resolution, special considerations were necessary to handle problems such as those that occur when two different-colored lines cross. This was handled by erasing conflicting parts of crossing lines. A new mode for graphic erasure was added to enable easier and faster erasure of lines. All graphic coordinate data input were standardized into a 2-byte/coordinate format. This permits the use of a full range of coordinate values to be sent with only a 7-bit code.

Basic communications and command sequence recognition are the responsibility of the SYSTC routines. Keyboard and line buffers are continually scanned for data, which are either displayed on the screen or are part of a command sequence. A special control code (ESC, 1B hex) is used to indicate the start of a command sequence. The command sequences include all special functions provided by the terminal, ranging from communications functions, such as baud rate and duplex mode, to all of the graphics functions that are performed by the terminal.

\section{CONCLUSIONS}

The original objective of this project was to develop a microprocessor-based operating system for the Intecolor $8001 \mathrm{G}$ that would provide a usable communications interface with a standard IBM APL system. It was hoped that significant enhancements could be made to the graphics capabilities of the terminal. Most of this has been accomplished; standard ASCII communications, buffered $\mathrm{I} / \mathrm{O}$ eliminating timing delays, addition of APL characters including overstrikes, and simplification of graphics routines have all been successfully implemented. Graphic function extensions such as object definition, real-time movement, and area shading, however, have not yet been completed. The effort required to develop the system was considerably more than was anticipated. Software development of this magnitude can be extremely expensive; approximate development costs of this system are now over $\$ 10,000$. This cost can only be justified if significant use of the software occurs.

\section{REFERENCES}

Dwyer, T. A., \& CRitchfield, M. Color graphics on the Compucolor 8051. Byte, 1978, 5, 32-39.

Gilman, L., \& Rose, A. J. APL an interactive approach. New York: Wiley, 1974.

Intelligent Systems Corporation. How to use the Intecolor 8001. Duluth, Ga: Author, 1976.

INTEL CORPORATION. Intel data catalog 1977. Bowers, Calif: Author, 1977.

Intel Corporation. MCS-86 system design kit. Bowers, Calif: Author, 1978.

Osborne, A. An introduction to microcomputers Volume IISome real products. Berkeley, Calif: Osborne, 1976.

RAO, G. R., \& Hewkis, J. 64-K dynamic Ram needs only one 5-volt supply to outstrip 16-K parts. Electronics, 1978, 20, 109-116.

TeKtronix, Inc. Tektronix 4013 computer display terminal users instruction manual. Beaverton, Ore: Author, 1973. 